\title{
Towards a Molecular Syndromology of the Epilepsies
}

During the past decade, there has been remarkable progress in elucidating the genetic basis of epilepsy disorders. This is due to new genome-wide technologies allowing us to screen for small copy number variants as well as for alterations of the DNA base sequence. This technical progress relieves clinicians from the urge of hypothesising about defined genetic causes and speculating about variations in a restricted number of genes or diverse chromosomal abnormalities. Furthermore, we have learnt a lot about the genetic architecture of the epilepsies and its pathophysiological principles. This especially holds true for rare diseases with epileptic seizures as a phenotypic feature. However, some important open questions remain unaddressed. For example, the complexity of genotype-phenotype correlations in these rare disorders is poorly understood [Steinlein, 2014], and the genetic background of common genetic epilepsies still remains unclear [Pal and Strug, 2014].

Despite these and other unresolved issues, one may ask whether our present knowledge allows us to outline some aspects of a molecular syndromology of the epilepsies. This themed issue of Molecular Syndromology tries to address diverse and frequent genetic aspects of epileptogenesis without the intention of being exhaustive.

The methodological progress of the past years is nicely illustrated by the example of epileptic encephalopa- thies. After defining the term epileptic encephalopathy and elucidating its complex relationship between epilepsy on the one side and cognitive and neurological impairment on the other, Helbig and Tayoun give a summary of novel insights. By focussing on the complexity of variable genotype-phenotype correlations within diseases underlying these genetic conditions, they outline that the same pathogenic variant in an epilepsy gene can cause epileptic encephalopathy as well as easy to treat or spontaneously resolving epilepsy without intellectual disability. Finally, it is discussed whether these allelic conditions represent a fluid continuum or distinct entities. Besides these aspects, they give a list of responsible genes pointing to disturbed functions of ion channels, neurotransmitter receptors, and presynaptic vesicle processing as main pathophysiological principles underlying epileptic encephalopathies as well as more benign forms of epilepsy.

Syrbe et al. illustrate the great phenotypic variability of a given genetic variant. They present a family with a novel SCN2A missense variant associated with phenotypes ranging from benign familial neonatal infantile seizures to Ohtahara syndrome. Gene expression studies in Xenopus laevis oocytes could not explain this variability of phenotypes associated with this specific variant taking into account that dysfunctional channel sub-

\section{KARGER}

E-Mail karger@karger.com

www.karger.com/msy
(C) 2016 S. Karger AG, Basel

1661-8769/16/0074-0169\$39.50/0 
units interact with other, not altered proteins in an intact organism. Thus, coexpression of all other (wild-type) channel components is necessary in order to simulate a more real situation and to elucidate the pathophysiology of these conditions. This is underlined by the study of Maljevic et al. on a novel KCNQ3 mutation in a large family with benign familial neonatal seizures.

In order to unravel genotype-phenotype correlations in diseases due to pathogenic variants of genes encoding ion channels or neurotransmitter receptor proteins, it may be interesting to also consider effects of autoantibodies against such proteins. The review by Lascano et al. compares cerebral autoimmunopathies associated with antibodies against LGI1 and NMDA receptors with channelopathies associated with pathogenic variants of LGI1 and NMDA receptor subunit genes. The genetically altered protein structures and the targets of autoantibodies are, however, not exactly the same. Furthermore, genetic variants represent constitutional conditions, whereas immunopathies due to autoantibodies interacting with the central nervous system are usually acquired. Thus, marked phenotypic similarities may seem unexpected.

Having detected numerous genetic variants underlying epilepsies and epileptic encephalopathies, the question arises on how we can facilitate diagnosis in everyday clinical practice. Møller et al. studied the diagnostic yield of a gene panel consisting of 46 epilepsy genes, most of them coding for ion channels, neurotransmitter receptors and proteins associated with or involved in synaptic processes. They found pathogenic variants in about a quarter of the analysed patients with the highest yield in neonatal-onset epilepsies and epileptic encephalopathies.

Epilepsy does not only result from dysfunctional signal transmission, but also from disturbed neuronal proliferation, migration, and organisation, which simultaneously predispose to impaired neurodevelopmental and psychiatric functioning. Parrini et al. give a comprehensive overview of these conditions describing the clinical MRI phenotypes and mentioning the underlying genes. They show that there is considerable variation in genotype-phenotype correlations. However, careful inspection of the MRI may allow for prioritising putatively causative genes in several conditions. Many of the involved genes code for proteins involved in mTOR signalling and other molecular pathways governing brain development and may occur as somatic mosaicism, especially in cases with mild or circumscribed morphological abnormalities.
Despite this increase of knowledge, the genetic basis of some well-known cerebral malformation syndromes still remains obscure. Aicardi syndrome is such an example, where females are affected by a typical trias consisting of infantile spasms, agenesis of corpus callosum and chorioretinal lacunae. Lund et al. applied wholeexome sequencing in 11 female patients and did not identify pathogenic or likely pathogenic variants. They suppose that causative genetic alterations may, for example, either be intronic or escape detection due to lowgrade mosaicism.

Of course, epilepsy does not only occur in the context of monogenic disorders. Numerous copy variants are associated with a higher likelihood of developing seizures, often but not always associated with other neurodevelopmental aspects, such as intellectual disability, autism spectrum disorders, and/or psychiatric diseases [Lal et al., 2015]. Since these microdeletions, duplications, and rearrangements usually affect several genes, genotype-phenotype correlations appear to be more complex than in monogenic disorders. This is exemplified by the study of Strehlow et al. analysing the occurrence of generalised epilepsy and myoclonic seizures in part resembling juvenile myoclonic epilepsy in $22 \mathrm{q} 11.2$ deletion syndrome.

Despite focussing on several frequent and rare causes of epilepsies, this themed issue can only refer to aspects of some genetic epilepsies and encephalopathies. Other disorders frequently associated with seizures, such as mitochondriopathies, inborn errors of metabolism, or neurodegenerative diseases have to remain unmentioned.

Finally, the aim of genetic testing is not only to gain certainty about an individual condition, but also to better understand its pathophysiologic background. The latter is focussed in this themed issue of Molecular Syndromology. It represents a prerequisite to investigate novel therapeutic prospects eventually leading to precision medicine approaches with the potential to significantly change the fate of patients. One promising example is pharmacological mTOR inhibition in tuberous sclerosis, which is able to suppress not only cellular proliferation in subependymal giant cell astrocytoma [Franz et al., 2013] and angiomyolipoma [Bissler et al., 2013] but most probably also epileptic activity [Krueger et al., 2013].

Thomas Dorn, Zurich

Johannes R. Lemke, Leipzig August 2016 
References

J, Kingswood JC, Radzikowska E, Zon nenberg BA, Frost M, et al: Everolimus for angiomyolipoma associated with tuberous sclerosis complex or sporadic lymphangioleiomyomatosis (EXIST-2): a multicentre, randomised, double-blind, placebo-controlled trial. Lancet 381:817-824 (2013).

-Franz DN, Belousova E, Sparagana S, Bebin EM, Frost M, et al: Efficacy and safety of everolimus for subependymal giant cell astrocytomas associated with tuberous sclerosis complex (EXIST-1): a multicentre, randomised, placebo-controlled phase 3 trial. Lancet 381: 125-132 (2013).
Krueger DA, Wilfong AA, Holland-Bouley K, Anderson AE, Agricola K, et al: Everolimus treatment of refractory epilepsy in tuberous sclerosis complex. Ann Neurol 74:679-687 (2013).

Lal D, Ruppert AK, Trucks H, Schulz H, de Kovel CG, et al: Burden analysis of rare microdeletions suggests a strong impact of neurodevelopmental genes in genetic generalised epilepsies. PLoS Genet 11:e1005226 (2015).

Pal DK, Strug LJ: The genetics of common epilepsies: common or distinct? Lancet Neurol 13: 859-860 (2014).

-Steinlein OK: Mechanisms underlying epilepsies associated with sodium channel mutations. Prog Brain Res 213:97-111 (2014). 